\title{
Fatores Determinantes da Retenção de Peso Pós-Parto em Lactantes Atendidas em Unidades de Saúde da Família do Município de Cuité/PB
}

\section{Determinant Factors of the Postpartum Weight Retention in Lactating Women Attended in Family Health Units of the city of Cuité/PB}

\section{RESUMO}

Introdução: Atualmente, a obesidade é considerada um problema de saúde pública e acomete cada vez mais mulheres em idade e estágio reprodutivo (gestação e retenção de peso pós-parto). Objetivo: Analisar os fatores determinantes da retenção de peso pós-parto em lactantes atendidas nas Unidades de Saúde da Família da zona urbana do Município de Cuité/PB. Métodos: Trata-se de um estudo de seguimento com 28 lactantes, com informações coletadas por meio de um questionário semiestruturado e da aferição de peso e altura no primeiro e quarto mês de lactação. Para análise dos dados foi utilizado o software estatístico SPSS for Windows. Resultados: Observa-se que as lactantes multíparas e que apresentaram ganho de peso excessivo durante a gestação retiveram mais peso, não se mostrando relação com o estado pré-gestacional. Nota-se maiores médias de retenção nas mães com maior idade, e renda, as quais obtiveram ganho de peso excessivo e tiveram parto cesário. Cabe destacar que a mulher que apresentou retenção de peso superior a dois quilos após o parto, teve um aumento no seu IMC, evidenciando uma possível relação entre a retenção de peso e a instalação de sobrepeso em mulheres. Conclusão: Com base nos resultados obtidos enfatiza-se a importância do cuidado nutricional contínuo com a mulher em idade reprodutiva, incluindo acompanhamento nutricional no período pré-gestacional, com melhorias na assistência pré-natal, e pós-parto, com a implantação de políticas públicas que proporcionem para a mulher subsídios necessários para a prevenção da obesidade.

DESCRITORES: retenção pós-parto, estado nutricional, ganho de peso, aleitamento materno, Saúde da mulher, lactação.

\begin{abstract}
Introduction: Obesity is currently considered a public health problem and affects an increasing number of women in reproductive age and stage (pregnancy and post-partum weight retention). Objective: To analyze determinant factors of postpartum weight retention in women cared for at the Family Health Units from urban area in the city of Cuité/PB. Methods: It is a follow-up study with 28 women, with information collected through a semi-structured questionnaire and weight and height tests performed in the first and fourth month of lactation. For data analysis, we used the SPSS statistical software for Windows. Results: It is observed that multiparous women and who had presented excessive weight gain during pregnancy retained more weight, showing no relation with the pre-pregnancy state. It is noticed a higher average of retention in mothers with higher age and income, who had presented an excessive weight gain and had caesarian section. It is important to point out that the woman who presented more than two kilos of weight retention after giving birth had an increase in her BMI, suggesting a possible connection between weight retention and overweight presence in women. Conclusions: Based on the results, it is emphasized the importance of continuous nutritional care to women in reproductive age, including nutritional monitoring in the pre-pregnancy period, with improvements in prenatal and postpartum care, and the implementation of public policies which provide necessary subsides for the prevention of obesity in women.
\end{abstract}

DESCRIPTORS: postpartum retention, nutritional state and weight gain, Breast Feeding, Women's Health, Lactation.

\footnotetext{
1 - Nutricionista. Pós-graduanda pela Residência Multiprofissional em Assistência Materno Infantil. Universidade Federal do Rio Grande do Norte, Santa Cruz, Rio Grande do Norte, Brasil.

2 - Nutricionista, Mestre, professora da Universidade Federal de Campina Grande, Campus: Cuité, Paraíba, Brasil.
} 
$\mathrm{A}$ tualmente, a obesidade é considerada um problema de saúde pública tanto nos países desenvolvidos quanto nos países em desenvolvimento, como o Brasil. Este novo panorama é fruto de uma série de modificações, inclusive nos padrões alimentares e de vida, sendo este fenômeno reconhecido como transição nutricional, que resulta de um rápido processo de mudanças nos perfis demográficos, econômicos, tecnológicos e de morbimortalidade nos últimos anos na população brasileira ${ }^{1,2}$.

Neste contexto, a Pesquisa de Orçamento Familiar (POF) realizada pelo Instituto Brasileiro de Geografia e Estatística (IBGE), no período de 2008-2009 demonstrou o crescimento da obesidade na Região Nordeste e em estratos sociais de maior vulnerabilidade econômica. Especificamente, na Região Nordeste, cerca de $45 \%$ das mulheres com mais de 20 anos de idade apresentaram excesso de peso e $15,2 \%$ obesidade ${ }^{3}$.

Diversos estudos destacam ainda que as mulheres em idade reprodutiva estão mais propensas ao desenvolvimento da obesidade, especialmente durante a gestação, visto que neste período ocorre um maior risco do ganho de peso em excesso ${ }^{4}$. Assim, estudos mais específicos confirmam que a gestação pode atuar como um efeito desencadeador ou agravante da obesidade em mulheres, em consequência da retenção de peso após o parto ${ }^{5,6}$.

A retenção de peso no período pós-parto, em geral, é uma condição determinada por múltiplos fatores, podendo destacar, o ganho de peso gestacional, o estado nutricional pré-gestacional, o estilo de vida e as condições socioeconômicas da mãe, apresentando assim, riscos para o desenvolvimento da obesidade ${ }^{7}$.

Neste sentido, evidencia-se a necessidade de conhecer melhor os fatores determinantes deste problema, visto a existência de poucos estudos no Brasil, especialmente, na Região Nordeste que abordem esta temática. Desta forma, este estudo busca descrever e estudar a retenção de peso pós-parto entre mulheres atendidas nas Unidades de Saúde da Família do Município de Cuité, localizado no semiárido Nordestino, Estado da Paraíba. A partir desta investigação, podem ser gerados subsídios para o planejamento e a implantação de ações de saúde pública, que visem à promoção e a atenção à saúde da mulher e a prevenção da obesidade. Neste contexto, o estudo teve como objetivo analisar os fatores determinantes da retenção de peso pós-parto em lactantes atendidas nas Unidades de Saúde da Família da zona urbana do município de Cuité/PB, estabelecendo relações entre as variáveis socioeconômicas, demográficas e sociais da mãe e do estado nutricional com a retenção de peso quatro meses pós-parto.

\section{Metodologia}

O presente estudo trata-se de um recorte de dados do projeto de pesquisa intitulado "Leite humano em diferentes estágios de lactação: estado antropométrico materno e composição nutricional no Município de Cuité" desenvolvido entre outubro de 2011 e abril de 2013, aprovado pelo Comitê de Ética em Pesquisa da Universidade Federal da Paraíba com CAEE: 0374.0.133.000-11. O referido projeto é um estudo de seguimento desenvolvido com o objetivo de analisar a composição nutricional do leite humano e o estado antropométrico de mães em aleitamento materno residentes no Município de Cuité no $1^{\circ} \mathrm{e} 4^{\circ}$ mês de lactação.

Para conferir maior homogeneidade a amostra de estudo, foram pré-estabelecidos critérios de inclusão (mães residentes na zona urbana do Município de Cuité, mulheres em processo de amamentação e parto único, e lactentes com mais de 15 dias de parto) e de exclusão (mães de recém-nascido retido em unidades de terapias intensivas neonatal ou berçários, crianças portadoras de anomalias congênitas que impeçam a amamentação, mães adolescentes e de bebês prematuros).

A captação da amostra realizou-se por meio de dois levantamentos de dados em todas as Unidades de Saúda da Família (USF) da zona urbana do Município de Cuité/PB, onde foram coletados o nome e o endereço de puérperas no primeiro mês de lactação e de todas as gestantes acompanhadas. A figura 1 apresenta o fluxograma explicativo dos procedimentos adotados para a captação de mães e a coleta de dados.

Após os dois momentos de levantamento e coleta de dados obteve-se um total de 35 mães, 
destas, 28 compuseram a amostra de estudo. Esta redução deveu-se a dois fatores importantes, a falta da informação referente ao peso pré-gravídico, visto que para 4 mulheres não foi possível registrar o peso pré-gestacional, em decorrência da ausência da informação na Unidade de Saúde (devido o início do pré-natal tardio), assim como a ausência do registro do peso no quarto mês de lactação, pois para outras 3 mulheres não foi registrado o peso no $4^{\circ}$. mês de lactação por óbito da criança, por dificuldade de encontrá-las em seu domicílio e por erros de procedimento das entrevistadoras de campo.

A coleta de dados aconteceu no domicílio das mães e foram realizadas duas visitas, sendo uma no primeiro e outra no quarto mês de lactação.
Na primeira visita realizada foi feita a apresentação do projeto, verificação das condições de inclusão e convite para participação na pesquisa. Comprovada as condições que tornavam a mãe apta, acontecia então a solicitação para a assinatura do Termo de Consentimento Livre e Esclarecido e a aplicação do instrumento de coleta de dados, sendo este um questionário semiestruturado para coleta de informações socioeconômicas e de características maternas, seguidos da aferição das medidas antropométricas da mãe, agendamento da visita referente ao quarto mês de lactação e, por fim, a coleta de leite humano.

$\mathrm{Na}$ segunda visita domiciliar, inicialmente, foi verificado se as mães ainda permaneciam em aleitamento materno para realização de uma

Figura 1: Fluxograma do trabalho de campo referente à captação da amostra para o projeto "Leite humano em diferentes estágios de lactação: estado antropométrico materno", 20112013. Fonte: Pesquisa, Leite humano, 2011-2013.

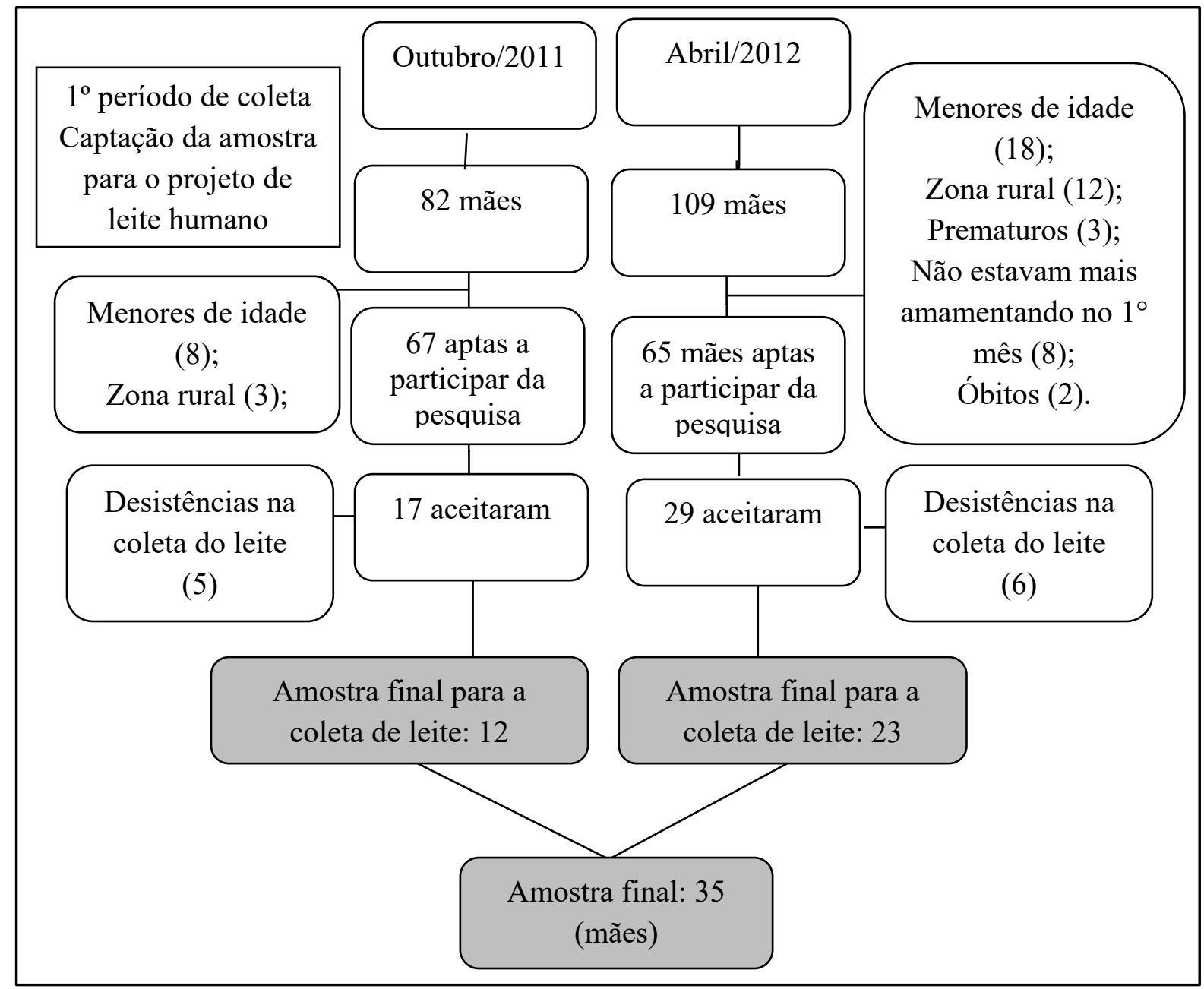


nova coleta de dados, como a aferição do peso no quarto mês de lactação e uma nova coleta de leite humano. As medidas antropométricas (peso e altura) foram aferidas segundo técnicas de aferição propostas por Duarte e Castellani, $2002^{\circ}$. As informações referentes ao peso pré-gestacional foram coletadas do cartão da gestante ou do relato da mãe. A segunda opção foi utilizada, apenas, quando esta informação não estava registrada no cartão da gestante.

As informações maternas coletadas foram transferidas para o meio digital do programa Microsoft Access da plataforma Windows e analisadas no software estatístico SPSS for Windows, utilizando técnicas descritivas e para análise de médias. Foi considerada como variável dependente a retenção de peso pós-parto e para construção de variáveis independentes foram adotadas informações sobre condições socioeconômicas, características demográficas e sociais da mãe, estado nutricional materno no período pré-gestacional, durante a gestação, no $1^{\circ}$ e $4^{\circ}$ mês de lactação, assim como a prática do aleitamento materno. As médias de retenção de peso pós-parto, segundo as variáveis independentes, foram comparadas utilizando-se o Test $-\mathrm{t}$. Utilizou-se também o Teste - $\mathrm{t}$ para comparação entre as médias do peso pré e pós-gestacional, assim como o IMC pré-gestacional e pós-gestacional das mulheres que apresentaram uma retenção de peso superior ou inferior a dois quilos.

Diversos indicadores antropométricos foram utilizados para análise de dados, o peso pré-gestacional, trata-se do peso materno anterior à gestação até a $13^{a}$ semana gestacional ${ }^{9}$. O IMC pré-gestacional e atual foi obtido por meio da razão entre o peso pré-gestacional em quilogramas $(\mathrm{Kg})$ e a altura em metros ao quadrado $\left(\mathrm{m}^{2}\right)$, a classificação do estado nutricional gravídico foi realizada segundo o indicador IMC por semana gestacional, devido às particularidades desta fase ${ }^{10}$. $O$ ganho de peso gestacional total foi determinado pela diferença entre o peso aferido na última consulta pré-natal e o peso pré-gestacional. Para avaliar a adequação de ganho de peso gestacional foram utilizados os limites superiores e inferiores de gan- ho de peso gestacional proposto pelo IOM, 2009'11, que recomenda ganhos de peso diferenciados de acordo com o estado nutricional pré-gestacional aferidos por meio do IMC.

Para verificar a retenção de peso pós-parto foi realizado o cálculo diferencial entre o peso pré-gestacional e o peso aferido no $1^{\circ} \mathrm{e} 4^{\circ}$ mês de lactação, e esta foi classificada em dois grupos, o primeiro que apresentou retenção de peso inferior a dois quilos e o segundo que apresentou retenção superior a dois quilos no quarto mês de lactação.

Com relação à etnia materna, caracterizou-se em branca e não-branca. A idade das lactantes foi dividida em dois grupos (20 a 30 anos/31 a 40 anos). Para a classificação da variável renda, foi observada a existência de famílias com uma renda mensal per capita de até $140,00^{12}$. No tocante a escolaridade, considerou-se mães de baixa escolaridade, aquelas que não possuíam escolaridade alguma ou tinham cursado o ensino fundamental incompleto/completo, para escolaridade alta foram agrupadas as lactantes que cursaram o ensino médio incompleto ou completo e que possuissem curso técnico profissionalizante ou curso superior. No que diz respeito a paridade, estas foram classificadas em multíparas (mais de um filho) ou primíparas (um único filho). A variável condição de atividade e ocupação foi categorizada em tem trabalho e não tem trabalho (dona de casa, procura trabalho e estudante). $O$ aleitamento materno foi classificado em aleitamento exclusivo, predominante e misto ${ }^{13}$.

A classificação de ganho de peso total durante a gestação foi categorizada em insuficiente, adequada e excessiva obedecendo aos limites para o ganho de peso gestacional de acordo com o estado nutricional inicial da gestante recomendados pelo Institute of Medicine, $2009^{11}$.

\section{Resultados}

O estudo foi constituído por uma amostra total de 28 mães, residentes na zona urbana do Município de Cuité-PB, em sua maioria adultas jovens de etnia não branca, que tiveram acesso aos serviços de saúde e dispunham de condições socioeconômicas e educacionais favoráveis. 
Ao analisar as prevalências e médias de retenção de peso pós-parto observou-se um percentual de $74,3 \%$ de mães com retenção de peso superior a dois quilos no quarto mês de lactação. As lactantes estudadas apresentaram uma retenção de peso no primeiro mês de até $12 \mathrm{~kg}$, com uma média de $2,67 \mathrm{Kg}( \pm 4,71)$ e no quarto mês observou-se uma redução do valor máximo de retenção de peso para até $10,7 \mathrm{Kg}$ e uma média de $2,03 \mathrm{Kg}( \pm 4,58)$.

A tabela 1 contém as médias de retenção de peso pós-parto, no quarto mês de lactação segundo os fatores demográficos e socioeconômicos, as características da gestação, do parto e da lactação. Então, observou-se que no tocante a renda ocorreu uma maior retenção de peso pós-parto em mulheres com renda acima da linha da pobreza, assim como entre mulheres brancas e que convivem em união estável. Ao avaliar a variável paridade observou-se que as multíparas apresentaram uma média de retenção de peso superior às primíparas, demonstrando diferença estatisticamente significativa, bem como as que apresentaram idade mais avançada.

No tocante as características antropométricas, o IMC pré-gestacional não apresentou relação com a retenção de peso pós-parto, ensejando que as mulheres que iniciaram a gestação eutróficas retiveram uma média de peso levemente superior de peso. Com relação ao ganho de peso gestacional, observou-se uma média de retenção de peso pós-parto superior em mulheres que obtiveram um ganho de peso excessivo, quando comparado àquelas que ganharam peso insuficiente ou adequado. As mulheres que passaram por procedimento de parto cesariano retiveram em média mais peso. Com relação a prática de aleitamento materno, as mães que estavam em aleitamento materno misto apresentaram uma menor retenção de peso, porém sem diferença estatisticamente significante.

A tabela 2 mostra a representação das médias de peso pré-gestacional e do quarto mês de lactação, bem como os valores médios de IMC nos períodos pré e pós-gestacionais, segundo os grupos de mulheres que apresentaram uma retenção de peso inferior e superior a dois quilos no quarto mês pós-parto.

Ao avaliar as médias do grupo de mulheres que apresentaram uma retenção superior a dois quilos, observou-se que estas, iniciaram com menor peso pré-gestacional e ao final do seguimento, no quarto mês de lactação, apresentaram um aumento médio no peso de 4,466 kg $\pm 2,12(p<0,05)$.

Observou-se ainda um comportamento inverso para aquelas que apresentaram retenção inferior a dois quilos, onde ocorreu uma redução do peso pré-gestacional para o peso pós-parto. Do mesmo modo, ao analisar o IMC verificou-se comportamentos semelhantes às alterações observadas nas variações de peso, com redução de média no IMC de $1,04 \mathrm{~kg} / \mathrm{m}^{2}( \pm 1,78)$, entre as que retiveram menos de dois quilos. No grupo de mulheres que retiveram mais que dois quilos, houve um aumento médio de $1,54 \mathrm{~kg} / \mathrm{m}^{2} \pm 0,56$ $(p<0,05)$.

\section{Discussão}

Com base nos resultados obtidos no presente estudo, notou-se que a incidência e as médias de retenção de peso encontraram-se semelhantes as identificadas em alguns estudos. Uma investigação realizada nos Municípios de Petrópolis e Queimados, no Rio de Janeiro ressaltou que $69 \%$ das mulheres avaliadas retiveram de 1 a $5 \mathrm{~kg}$ ou mais, transcorridos seis meses após o parto ${ }^{14}$. Com relação às médias, Vasconcelos et al, $2014^{15}$ encontraram uma média de retenção de $2,39 \mathrm{Kg}( \pm 5,86)$, assim como, em uma outra pesquisa realizada por Kac et al, $2004^{16}$, em que a média identificada foi de $2,5 \mathrm{~kg}$, aos nove meses pós-parto.

No tocante a renda observou-se uma concordância com os resultados expostos por Rebelo et al, $2010^{7}$, demonstrando que existiu uma maior retenção de peso em mulheres que convivem com renda familiar, mais favorável. Alguns estudos agrupados em uma revisão de literatura não demonstram relação entre a retenção e a escolaridade materna ${ }^{17}$, o que também foi observado nesta investigação. 
Tabela 1: Representação das médias de retenção de peso pós-parto, no quarto mês de lactação segundo fatores demográficos, socioeconômicos, características da gestação, do parto e da lactação, de lactantes residentes na zona urbana do município de Cuité, PB, 2011/2013.

\begin{tabular}{|c|c|c|c|}
\hline Variáveis & Médias & $\begin{array}{c}\text { Desvio padrão } \\
( \pm)\end{array}$ & $p$ \\
\hline \multicolumn{4}{|l|}{ Renda } \\
\hline Abaixo da linha da pobreza & 1,30 & 6,11 & \multirow[t]{2}{*}{0,633} \\
\hline Acima da linha da pobreza & 2,28 & 4,10 & \\
\hline \multicolumn{4}{|l|}{ Escolaridade } \\
\hline Baixa & 1,91 & 4,00 & \multirow[t]{2}{*}{0,944} \\
\hline Alta & 2,06 & 4,81 & \\
\hline \multicolumn{4}{|l|}{ Ocupação } \\
\hline Tem trabalho & 2,01 & 4,02 & \multirow[t]{2}{*}{0,987} \\
\hline Não tem trabalho & 2,04 & 5,03 & \\
\hline \multicolumn{4}{|l|}{ Presença do companheiro } \\
\hline Sim & 2,73 & 3,21 & \multirow[t]{2}{*}{0,355} \\
\hline Não & $-1,18$ & 8,31 & \\
\hline \multicolumn{4}{|l|}{ Etnia } \\
\hline Branca & 2,44 & 3,87 & \multirow[t]{2}{*}{0,792} \\
\hline Não-branca & 1,90 & 4,87 & \\
\hline \multicolumn{4}{|l|}{ Idade } \\
\hline $20-30$ & 1,63 & 4,69 & \multirow[t]{2}{*}{0,382} \\
\hline $31-40$ & 3,51 & 4,17 & \\
\hline \multicolumn{4}{|l|}{ Paridade } \\
\hline Primíparas & 0,07 & 5,27 & \multirow[t]{2}{*}{$0,048^{*}$} \\
\hline Multíparas & 3,50 & 3,46 & \\
\hline \multicolumn{4}{|l|}{ IMC pré-gestacional } \\
\hline Eutrofia & 2,09 & 4,57 & \multirow{2}{*}{0,923} \\
\hline Sobrepeso & 1,90 & 4,90 & \\
\hline \multicolumn{4}{|l|}{ Ganho de peso gestacional } \\
\hline Insuficiente & $-2,23$ & 5.93 & \multirow{2}{*}{$0,04^{*}$} \\
\hline Adequado & 2,91 & 1,55 & \\
\hline Excessivo & $\begin{array}{l}2,91 \\
4,98\end{array}$ & $\begin{array}{l}1,35 \\
3,12\end{array}$ & 0,06 \\
\hline \multicolumn{4}{|l|}{ Tipo de parto } \\
\hline Normal & 1,21 & 5,13 & \multirow[t]{2}{*}{0,353} \\
\hline Cesário & 2,85 & 3,97 & \\
\hline \multicolumn{4}{|l|}{ Tipo de aleitamento } \\
\hline Exclusivo & 2,38 & 3,92 & \multirow[t]{2}{*}{0,638} \\
\hline Misto & 1,19 & 5,88 & \\
\hline
\end{tabular}


Tabela 2: Representação das médias de peso e IMC pré e pós-gestacional ( $4^{\circ}$ mês) dos grupos que retiveram peso maior e menor que dois quilos no pós-parto, de lactantes residentes na zona urbana do município de Cuité, PB, 2011/2013.

\begin{tabular}{c|c|c}
\hline Variáveis & $\begin{array}{c}\text { Retenção inferior a } \\
\text { dois quilos }(\mathbf{k g}) / \mathbf{D P}\end{array}$ & $\begin{array}{c}\text { Retenção superior a dois quilos } \\
(\mathbf{k g}) / \mathbf{D P}\end{array}$ \\
\hline Peso pré-gestacional $(\mathrm{kg})$ & $63,00( \pm 8,89)$ & $58,88( \pm 8,20)$ \\
\hline Peso do $4^{\circ}$ mês $(\mathrm{kg})$ & $60,66( \pm 9,85)$ & $63,35( \pm 8,61)$ \\
\hline $\boldsymbol{p}$ & 0,14 & $\mathbf{0 , 0 0 *}$ \\
\hline IMC pré-gestacional $\left(\mathrm{kg} / \mathrm{m}^{2}\right)$ & $24,66( \pm 2,09)$ & $22,89( \pm 3,51)$ \\
\hline IMC pós-gestacional $\left(\mathrm{kg} / \mathrm{m}^{2}\right)$ & $23,22( \pm 2,70)$ & $24,44( \pm 3,77)$ \\
\hline $\boldsymbol{p}$ & $\mathbf{0 , 0 9 ^ { * }}$ & $\mathbf{0 , 0 0 *}$ \\
\hline
\end{tabular}

${ }^{*} p<0,05$.

Poucos estudos mostram a relação da etnia materna e da convivência com o companheiro e a retenção de peso no pós-parto, entretanto, Jordão e Kac $2005^{18}$ indicam uma menor chance de reter peso pós-parto entre mulheres da cor negra e solteiras.

As multíparas apresentaram uma maior retenção de peso corroborando com alguns resultados existentes na literatura ${ }^{18,19}$. No presente estudo ressalta-se uma retenção de peso pós-parto superior em mulheres com idade mais avançada, como demonstrado em outros estudos ${ }^{18,20}$. Sendo assim, torna-se possível estabelecer uma relação entre a multiparidade e a idade mais avançada da mulher, como fatores associados com uma maior retenção de peso, que pode estar associada há um histórico de ganho de peso excessivo e de retenção de peso pós-parto em uma gestação anterior.

Com relação às medidas antropométricas, o Índice de Massa Corpórea (IMC) pré-gestacional, as mulheres que iniciaram a gestação eutróficas retiveram uma média levemente superior de peso, corroborando com os resultados apresentados por Vasconcelos $2007^{21}$, que demonstram um risco aumentado de retenção superior a 7,5 quilos entre as mulheres com IMC pré-gravídico menor que $25 \mathrm{~kg} / \mathrm{m}^{2}$. Argumenta-se que a mulher que inicia a gestação com excesso de peso é melhor amparada pelos serviços de saúde com o objetivo de controlar o ganho de peso ao longo da gestação e oferecer ao bebê os nutrientes necessários para seu pleno desenvolvimento ${ }^{14}$.

Sendo assim, os resultados sugerem que a prevenção da retenção de peso deve estar voltada para todas as gestantes, inclusive aquelas com diagnóstico de eutrofia no período pré-gestacional, tendo em vista que estas retiveram peso após o parto e podem manter tal padrão em gestações subsequentes.

Alguns estudos confirmam a hipótese encontrada neste estudo com relação ao ganho de peso excessivo durante a gestação. Uma investigação do tipo coorte com 104 gestantes recrutadas em uma Unidade Básica de Saúde, no Município do Rio de Janeiro, demonstrou que a retenção de peso foi maior entre as mulheres que apresentaram ganho de peso gestacional excessivo, em comparação às mulheres com ganho de peso gestacional adequado ou insuficiente ${ }^{7}$, resultados semelhantes também foram encontrados por Colebrusco, $2010^{22}$.

No tocante ao tipo de parto, no presente estudo foi encontrado um resultado semelhante ao apresentado por Vasconcelos, et al., $2014^{15}$ e que difere dos expostos por Rebelo, $2010^{7}$.

As lactantes que estavam em aleitamento 
materno misto apresentaram uma média inferior de retenção de peso quando comparadas aquelas que estavam em aleitamento materno exclusivo, corroborando com os resultados expostos por Vasconcelos $2007^{21}$ que não identificaram influência do tipo de aleitamento materno sobre a retenção no pós-parto.

Importa destacar que a mulher em lactação apresenta um aumento no apetite e os tipos de alimentos que estão sendo consumidos neste período pós-parto podem apresentar relação com a retenção de peso. Um estudo realizado por Martins e Benicio, 201122 no Município de São Paulo, demonstrou que um consumo aumentado de gordura saturada e de alimentos processados apresenta relação estatisticamente significativa com a retenção de peso, 15 dias após o parto. Outro estudo realizado por Lacerda et al., $2007^{23}$ demonstrou a relação entre consumo excessivo de energia e de gorduras saturadas durante a gestação, com a retenção no período pós-parto.

Neste estudo não foi contemplado nenhum tipo de inquérito sobre o consumo alimentar da lactante, o que sugere a importância desta investigação em estudos futuros, buscando elucidar a influência do padrão alimentar durante e após a gestação sobre a retenção de peso pós-parto.

Diante de todos os fatores que foram discutidos é importante ressaltar que o ganho de peso durante a gestação é um fator passível a modificações, portanto, quando identificada alguma inadequação (ganho insuficiente ou excessivo), tornam-se necessárias intervenções no que diz respeito ao melhor planejamento deste ganho de peso, visando um maior controle e acompanhamento, proporcionando assim benefícios para a saúde da mulher e desenvolvimento da criança no período gestacional.

Estas inadequações do ganho de peso gestacional devem ser identificadas durante as consultas de pré-natal, permitindo assim a possibilidade de realização das consultas compartilhadas, sendo estas realizadas em conjunto pela Equipe de Saúde da Família e o Núcleo de Apoio a Saúde da Família (NASF), contando com a visão ampla de vários profissionais incluindo o nutricionista, possibilitando assim intervenções mais eficazes, através da identificação dos condicionantes do problema ${ }^{24}$.

Esta preocupação com a saúde da mulher não deve estar voltada apenas para o período gestacional e puerperal, sendo necessária uma atenção integral, que está sendo proposta pela Rede Cegonha visando assegurar à mulher o direito ao planejamento reprodutivo e à atenção humanizada na gravidez, parto e puerpério, bem como o acompanhamento à criança, nos 2 primeiros anos de vida ${ }^{25}$. Desta forma, é indispensável o cuidado contínuo com a mulher incluindo ações que visem proporcionar o controle de peso e o estabelecimento de hábitos alimentares saudáveis, valorizando a cultura alimentar, exercendo influência futura na saúde da mãe e do bebê.

No que diz respeito às variações de peso nos períodos pré-gestacional e pós-natal, não foi encontrado nenhum estudo que relacionasse as diferenças de peso e o IMC no período pré-gestacional e pós-natal, segundo a quantidade de quilos retidos no pós-parto. Os resultados apresentados por Vasconcelos $2007^{21}$ e Cabral $2010^{26}$ mostraram que ocorreu um acréscimo do IMC pré-gestacional para o pós-gestacional.

\section{Conclusão}

A retenção de peso pós-parto configura-se, atualmente, como uma condição de risco aumentada para a implantação de sobrepeso e obesidade em mulheres com idade reprodutiva. No presente estudo foi observado que ocorreu retenção de peso pós-parto na maioria das mulheres com média de dois quilos, no quarto mês de lactação. Destaca-se, ainda, maiores médias de retenção nas mães com maior idade, renda acima da linha da pobreza, que convivem com o companheiro, que se consideram de cor branca, que apresentaram ganho de peso 
excessivo, tiveram parto cesário e estão em aleitamento materno exclusivo. Observou-se ainda que a retenção de peso de aproximadamente dois quilos foi suficiente para influenciar no estado nutricional da mulher no quarto mês de lactação, evidenciando sua relação com a instalação do sobrepeso.

Com base no exposto torna-se necessário à implementação de políticas públicas voltadas para mulher em idade reprodutiva que englobem ações de promoção de hábitos alimentares e de vida saudáveis, e prevenção de agravos da saúde, como obesidade, desnutrição ou instalação de deficiências nutricionais.

\section{REFERÊNCIAS}

1. Filho MB, Souza AI, Miglioli TC, Santos MC. Anemia e obesidade: um paradoxo da transição nutricional brasileira. Cad. de Saúde Públ. 2008; 24 (2):247-257.

2. Malta DC, Cezário AC, Moura L, Neto OLM, Junior JBS. A construção da vigilância e prevenção das doenças crônicas não transmissíveis no contexto do Sistema Único de Saúde. Epidemiologia e Serviços de Saúde. 2006;15 (3):47-65.

3. IBGE - Instituto Brasileiro de Geografia e Estatística. Pesquisa de Orçamentos Familiares 2008-2009 - POF. Rio de Janeiro, 2010.

4. Lacerda EMA, Leal MC. Fatores associados com a retenção e o ganho de peso pós-parto: uma revisão sistemática. Rev Bras Epidemiologia. 2004; 7(2):187-200.

5. Stulbach TE, Benício MHD, Andreazza R, Kono S. Determinantes do ganho ponderal excessivo durante a gestação em serviço público de pré-natal de baixo risco. Rev Bras de Epidemiologia, 2007; 10(1):99-108, 2007.

6. Padilha PC, Saunders C, Machado RCM, Silva CL, Bull A, Sally EOF, Accioly E. Associação entre o estado nutricional pré-gestacional e a predição de riscos de intercorrências gestacionais. Rev Bras de Ginecologia e Obstetrícia. 2007; 29(10):511-518.

7. Rebelo F, Castro MBT, Dutra CL, Schlussel MM, Kac G. Fatores associados à retenção de peso pós parto em uma coorte de mulheres, 2005-2007. Rev Bras de Saúde Materno Infant., 10(2):219-227, 2010.

8. Duarte AC, Castellani FR. 1ed.. Rio de Janeiro: Axcel Books, 2002.

9. Kac G, Sichieri R, Gigante DP. Epidemiologia Nutricional. Rio de Janeiro: Fiocruz/Atheneu, 2007. 580 p.

10. Brasil. Vigilância alimentar e nutricional - Sisvan: orientações básicas para a coleta, processamento, análise de dados e informação em serviços de saúde, 2004. 120 p.

11. Institute Of Medicine, Weight Gain During Pregnancy: Reexamining the Guidelines. The National Academy Press, 2009.

12. Brasill. Ministério do Desenvolvimento Social e Combate a Fome. Secretaria Nacional de Renda de Cidadania Instrução Operacional n 47 de 23 de agosto de 2011:Orienta sobre os procedimentos para correção das informações das famílias habilitadas para Programa Bolsa Família, com dados desatualizados na base do Cadastro Único. 2011. 5 p.

13. Brasil. Ministério da Saúde. Secretaria de Atenção à Saúde. Departamento de Atenção Básica. Saúde da criança: Nutrição infantil: aleitamento materno e alimentação complementar / Ministério da Saúde, Secretaria de Atenção à Saúde, Departamento de Atenção Básica. - Brasília: Editora do Ministério da Saúde, 2009.

14. Nogueira, JL. Fatores associados à retenção de peso em mulheres no pós-parto em municípios do Rio de Janeiro. 2014. 167 F. Tese (Doutorado de Epidemiologia em Saúde Pública). Escola Nacional de Saúde Publica - Rio de Janeiro, 2014.

15. Vasconcelos CMCS, Costa FS, Almeida PC, Junior EA Sampaio HACS. Fatores de risco associados à retenção de peso seis meses após o parto. Rev Bras de Ginecologia e Obstetrícia. 2014; 36(5):222-227.

16. Kac G, Benicio MHDA, Velásquez-melénde, G, Valente J.D. Nine months postpartum weight retention predictors for Brazilian Women. Public Health Nutrition. 2004; 7(5):621-628.

17. Lacerda EMA, Leal MC. Fatores associados com a retenção e o ganho de peso pós-parto: uma revisão sistemática. Rev Bras Epidemiologia. 2004; 7(2),187-200.

18. Jordão ISC, Kac G. Determinantes da retenção de peso pós-parto segundo a cor da pele em mulheres do Rio de Janeiro, Brasil. Rev Panamerican Salud Publica. 2005; 18(6): 403-411.

19. Coitinho DC, Sichieri R, Benicio MHDA. Obesity and weight change related to parity and breast-feeding among parous womem in Brazil. Public Health Nutrition. 2001; 4(1): 865-870. 
20. Amaral FB. Determinantes da retenção de peso após o parto em mulheres atendidas nos serviços públicos de pré-natal do Distrito Federal. 2006. 142 f. Dissertação (Mestrado em Nutrição Humana) - Universidade de Brasília, Brasília, 2006.

21. Vasconcelos CMCS. Fatores associados com a retenção de peso no pós-parto. 2007. 153 F. Dissertação (Mestrado Acadêmico em Saúde Pública) - Universidade Estadual Do Ceará, Fortaleza, 2007.

22. Martins APB, Benicio MHD. Influência do consumo alimentar na gestação sobre a retenção de peso pós-parto. Rev de Saúde Pública. 2011; 45(5):870-877, 2011.

23. Lacerda EMA, Kac G, Cunha CB, Leal MC. Consumo alimentar na gestação e no pós-parto segundo cor da pele no município do Rio de Janeiro. Rev de Saúde Pública. 2007; 41 (6): 985-994.

24. Brasil. Ministério da Saúde. Secretaria de Atenção à Saúde. Departamento de Atenção Básica. Núcleo de Apoio à Saúde da Família, Brasília: Ministério da Saúde, 2014. 112 p
25. Brasil. Ministério da Saúde. Secretaria de Atenção à Saúde da mulher. Departamento de Atenção Básica. Manual instrutivo das ações de alimentação e nutrição na Rede Cegonha , 2013. 32 p.

26. Cabral MLGF. Consumo de proteínas na gestação e retenção de peso no pós-parto. 2010. 48 f. Trabalho de investigação - Faculdade de ciências da nutrição e alimentação, Universidade do Porto, Porto, 2010

\section{CORRESPONDÊNCIA}

Rayane Santos de Lucena

Rua Praça Tequinha Farias, número 78, apartamento 9 Santa Cruz - RN.

Email: rayanenutricao@hotmail.com 